\title{
Synthesis of a novel D-A type photoelectrical material based on fluorene
}

\author{
YAN Hewei ${ }^{1, a}$, TANG Zebiao ${ }^{1, b}$, SHEN Huayin ${ }^{1, c}$, SUN Xiaoxia ${ }^{1, d^{*}}$ \\ ${ }^{1}$ Jiangxi Key Laboratory of Organic Chemistry, Jiangxi Science and Technology Normal University, \\ Nanchang 330013, People's Republic of China \\ ayanhewei1990@163.com; btangzebiao88@163.com; 'shenhuayin49565880@163.com; \\ dsunxiaoxia77@126.com
}

Keywords: D-A type molecules; photoelectrical matrerial; Knevenagel reaction

\begin{abstract}
Novel D-A type conjugated organic molecules composed of central fluorene units and cyanoacetic acid terminal groups have been designed and constructed. The article was aimed at improving the solubility of small molecules by introducing aliphatic chains, and improving the optical and electrical properties of small molecules by introducing cyanoacetic acid. The target compounds would be promising candidates for organic light-emitting diodes.
\end{abstract}

\section{Introduction}

During the past decade, organic conjugated molecules with donor-acceptor architectures have attracted much academic and technological research attention, due to their potential electronics and optoelectronics applications such as organic field-effect transistors (OFETs), organiclightemitting diodes (OLEDs) and organic photovoltaics (OPVs) ${ }^{1-3}$ Conjugated organic molecules have great advantages on higher purity, fixed molecular weight and their clear molecular structure compared with organic polymers, which will receive a significant amount of interest by researchers in the coming years. ${ }^{4}$ Moreover, the donor-acceptor materials broadly provide large $\pi$-electron delocalization over the molecules, leading to desirable semiconductor properties for electrical and optical applications.

In order to improve the property of these materials, exploring new synthesis and investigation of conjugated molecules is significance, which have highly capable of accepting both electrons and holes while exhibiting high luminescence quantum yield. As an electron donor, fluorene has biphenyl structure with rigid plane and large $\pi$-conjugated system, plays an important role in the donor-acceptor organic molecules. Fluorene and its derivatives were extensively investigated and have been become active highlight in recent years, because of their special structures, various functional groups and being easily modified endowing their wide potential application in photoelectrical materials. $^{5}$

In this article, we design and synthesize new compounds (2Z,2'E)-3,3'-(5,5'-(9,9-dioctyl-9H -fluorene-2,7-diyl)bis(thiophene-5,2-diyl))bis(2 -cyanoacrylic acid), based on fluorene units at the center and introduced cyanoacetic acid group and thiophene to improve electronic and optical properties of organic conjugated molecules. The target compounds would be promising candidates for organic light-emitting diodes.Optical properties, electrical Properties and thermodynamic properties will be studied in future work.

\section{Experimental Section}

Characterization: ${ }^{1} \mathrm{H}$ NMR and ${ }^{13} \mathrm{C}$ NMR spectra were collected on a Bruker $\mathrm{AM}-400 \mathrm{WB}$ spectrometer in chloroform-d or DMSO as solvent and tetramethylsilane (TMS) as the internal standard. Materials: Diethyl ether and tetrahydrofuran (THF) were dried over sodium benzophenone and distilled under argon atmosphere before use. $\mathrm{CHCl}_{3}$ and $\mathrm{DMF}$ were dried over $\mathrm{CaH}_{2}$ and then distilled under nitrogen atmosphere and deoxygenated by purging with nitrogen for 30 min before use in monomer synthesis. Other chemicals were purchased from Acros and used without further purification. 


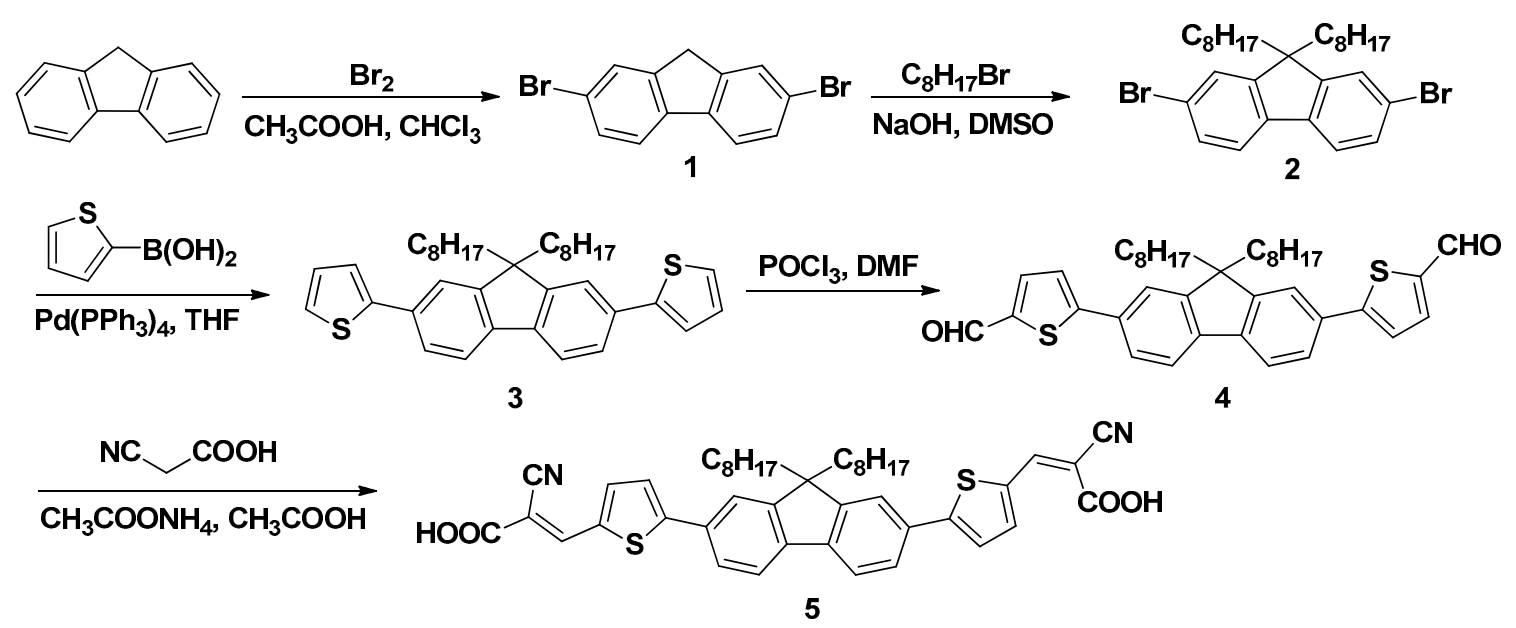

Scheme 1. The synthesis route of (2Z,2'E)-3,3'-(5,5'-(9,9-dioctyl-9H-fluorene-2,7diyl)bis(thiophene-5,2-diyl))bis(2 -cyanoacrylic acid) (5)

In our previous report, the compound $\mathbf{4}$ was synthesized via the Vilsmeier-Haack reaction as illustrated in Scheme 1. First, the reaction of 9H-fluorene with Bromine and acetic acid in $\mathrm{CHCl} 3$ give 1 in $89 \%$ yield. Then 2,7-dibromo-9H-fluorene and 1-bromooctane in DMSO with a moderate condition to give 2 in $96 \%$ yeild. In the next step, 2,7-dibromo-9,9-dioctyl-9H-fluorene reacted with 2.5 equiv. of thiophen-2-ylboronic acid to afford 3 in $86 \%$ yield. The target compound 4 was reacted using DMF and $\mathrm{POCl}_{3}$ to give a yield of $80 \%$.

Synthesis of 2,7-dibromo-9H-fluorene (1)

Bromine(4.03 g, $25.2 \mathrm{mmol})$ was slowly added using a syringe to the solution of 9H-fluorene $(2.00 \mathrm{~g}, 12.0 \mathrm{mmol})$ and acetic acid $(1 \mathrm{ml})$ in $\mathrm{CHCl}_{3}(30 \mathrm{ml})$. After stirring the mixture at room temperature for $12 \mathrm{~h}$, a precipitate was obtained and filtered, washed with distilled water and methanol. The product was obtained as white crystals. Yield: $3.46 \mathrm{~g}(89 \%) .{ }^{1} \mathrm{H}$ NMR $(400 \mathrm{MHz}$, $\left.\mathrm{CDCl}_{3}, \mathrm{ppm}\right): \delta=7.66(\mathrm{~s}, 2 \mathrm{H}), 7.58(\mathrm{~d}, \mathrm{~J}=7.6 \mathrm{~Hz}, 2 \mathrm{H}), 7.50(\mathrm{~d}, \mathrm{~J}=8.0 \mathrm{~Hz}, 2 \mathrm{H}), 3.84(\mathrm{~s}, 2 \mathrm{H}) .{ }^{13} \mathrm{C}$ NMR (100 MHz, $\left.\mathrm{CDCl}_{3}\right): \delta 44.79,139.82,130.03,128.29,121.21,36.56$.

Synthesis of 2,7-dibromo-9,9-dioctyl-9H-fluorene (2)

2,7-dibromo-9H-fluorene (2.80 g, $8.64 \mathrm{mmol})$ and 1-bromooctane $(3.67 \mathrm{~g}, 19.0 \mathrm{mmol})$ were dissolved in DMSO $(40 \mathrm{ml})$ and stirred for $30 \mathrm{~min}$ at room temperature, A sodium hydroxide solution $(10 \mathrm{ml}, \mathrm{v}: 50 \%)$ was slowly added and then stirred for $24 \mathrm{~h}$ at room temperature. The reaction mixture was poured into water and extracted by acetic ether. The organic layer separated and dried over anhydrous magnesium sulfite. The crude product was purified using column chromatography with petroleum ether as the eluent. The product was obtained as off-white solid. Yield: $4.55 \mathrm{~g}(96 \%) .{ }^{1} \mathrm{H}$ NMR (400 MHz, $\left.\mathrm{CDCl}_{3}, \mathrm{ppm}\right): \delta=7.53-7.51(\mathrm{~m}, 2 \mathrm{H}), 7.47-7.45(\mathrm{~m}, 4 \mathrm{H})$, $1.95-1.91(\mathrm{~m}, 4 \mathrm{H}), 1.25-1.05(\mathrm{~m}, 20 \mathrm{H}), 0.84(\mathrm{t}, \mathrm{J}=7.0 \mathrm{~Hz}, 6 \mathrm{H}), 0.59(\mathrm{~s}, 4 \mathrm{H}) .{ }^{13} \mathrm{C} \mathrm{NMR}(100 \mathrm{MHz}$, $\left.\mathrm{CDCl}_{3}\right): \delta 152.57,139.21,130.17,126.78,121.61,121.15,55.60,40.19,31.80,29.90,29.21,28.51$, $23.65,22.70,14.27$.

\section{Synthesis of 2,2'-(9,9-dioctyl-9H-fluorene-2,7-diyl)dithiophene (3)}

A mixture of 2,7-dibromo-9,9-dioctyl-9H-fluorene (4.00 g, $7.30 \mathrm{mmol})$, thiophen-2-ylboronic acid $(2.34 \mathrm{~g}, 18.5 \mathrm{mmol})$ and $\mathrm{Pd}\left(\mathrm{PPh}_{3}\right)_{4}(0.92 \mathrm{~g}, 0.80 \mathrm{mmol})$ in dry THF $(150 \mathrm{ml})$ and a $2.0 \mathrm{M}$ $\mathrm{K}_{2} \mathrm{CO}_{3}$ aqueous solution $(20 \mathrm{ml})$ was stirred for $24 \mathrm{~h}$ at $80{ }^{\circ} \mathrm{C}$ under $\mathrm{N}_{2}$. After cooling the mixture to the room temperature, the solvent was removed, and the product was purified using column chromatography on a silica gel with petroleum ether. The product was obtained as green oil. Yield: $3.51 \mathrm{~g}(86 \%) .{ }^{1} \mathrm{H}$ NMR (400 MHz, $\left.\mathrm{CDCl}_{3}, \mathrm{ppm}\right): \delta=7.61(\mathrm{~s}, 2 \mathrm{H}), 7.58(\mathrm{~s}, 2 \mathrm{H}), 7.39$ (d, J=3.6 Hz, 2H), $7.29(\mathrm{~d}, \mathrm{~J}=5.2 \mathrm{~Hz}, 2 \mathrm{H}), 2.04-2.01(\mathrm{~m}, 4 \mathrm{H}), 1.25-1.05(\mathrm{~m}, 20 \mathrm{H}), 0.89(\mathrm{t}, \mathrm{J}=7.0 \mathrm{~Hz}, 6 \mathrm{H}), 0.71$ (s, $4 \mathrm{H}) .{ }^{13} \mathrm{C}$ NMR $\left(100 \mathrm{MHz}, \mathrm{CDCl}_{3}\right): \delta 152.04,145.37,140.54,133.69,128.37,125.34,124.73$, $123.18,120.57,120.33,55.62,40.78,32.18,30.38,29.58,24.19,23.00,14.51$.

\section{Synthesis of 5,5'-(9,9-dioctyl-9H-fluorene-2,7-diyl)bis(thiophene-2-carbaldehyde) (4)}

2,2'-(9,9-dioctyl-9H-fluorene-2,7-diyl)dithiophene (0.5 g, $0.9 \mathrm{mmol})$ and DMF (1.39 $\mathrm{ml})$ was dissolved in 1,2-dichloroehane $(20 \mathrm{ml})$ and $\mathrm{POCl}_{3}(1.66 \mathrm{ml})$ was carefully added through a dropping funnel, keeping the reaction temperature below $5{ }^{\circ} \mathrm{C}$. After the complete addition of $\mathrm{POCl}_{3}$, the 
reaction solution was was stirred for $12 \mathrm{~h}$ at reflux. Then the mixture was poured into a cooled saturated sodium bicarbonate solution, neutralized with $\mathrm{Na}_{2} \mathrm{CO}_{3}$ solution, extracted with dichloromehane and washed with brine. The organic layer separated and dried over anhydrous magnesium sulfite. The crude product was purified using column chromatography with petroleum ether/dichloromethane $(2: 1, \mathrm{v} / \mathrm{v})$ as the eluent. The product was obtained as yellow solid. Yield: $0.44 \mathrm{~g},(80 \%) .{ }^{1} \mathrm{H}$ NMR (400 MHz, $\left.\mathrm{CDCl}_{3}, \mathrm{ppm}\right): \delta=9.91(\mathrm{~s}, 2 \mathrm{H}), 7.77(\mathrm{~d}, \mathrm{~J}=4.3,4 \mathrm{H}), 7.71(\mathrm{~d}$, $\mathrm{J}=8.0 \mathrm{~Hz}, 2 \mathrm{H}), 7.64(\mathrm{~s}, 2 \mathrm{H}), 7.50(\mathrm{~s}, 2 \mathrm{H}), 2.05-2.02(\mathrm{~m}, 4 \mathrm{H}), 1.13-1.05(\mathrm{~m}, 20 \mathrm{H}), 0.78(\mathrm{t}, \mathrm{J}=6.6$ $\mathrm{Hz}, 6 \mathrm{H}), 0.66(\mathrm{~s}, 4 \mathrm{H}) .{ }^{13} \mathrm{C} \mathrm{NMR}\left(100 \mathrm{MHz}, \mathrm{CDCl}_{3}\right): \delta 182.72,154.79,152.26,142.25,141.64$, 137.44, 132.42, 125.73, 124.06, 120.78, 120.71, 55.56, 40.21, 31.74, 29.87, 29.14, 23.76, 22.58, 14.03.

Synthesis of (2Z,2'E)-3,3'-(5,5'-(9,9-dioctyl-9H-fluorene-2,7-diyl)bis(thiophene-5,2-diyl))bis (2-cyanoacrylic acid) (5)

A mixture of 5,5'-(9,9-dioctyl-9H-fluorene-2,7-diyl)bis(thiophene-2-carbaldehyde) (4) (0.5 g, $0.82 \mathrm{mmol}$ ), 2-cyanoacetic acid ( $0.28 \mathrm{~g}, 3.27 \mathrm{mmol})$, ammonium acetate ( $0.25 \mathrm{~g}, 3.27 \mathrm{mmol})$ in AcoH $(50 \mathrm{~mL})$ was stirred for $12 \mathrm{~h}$ at $120{ }^{\circ} \mathrm{C}$ under $\mathrm{N}_{2}$. After it was cooled to room temperature, the reaction mixture was poured into ice water then filtered. The product was obtained as red solid. Yield: $0.52 \mathrm{~g}(85 \%) .{ }^{1} \mathrm{H}$ NMR (400 MHz, DMSO) $\delta 8.52(\mathrm{~s}, 2 \mathrm{H}), 8.05$ (d, J = 3.6 Hz, 2H), 7.94 (d, $\mathrm{J}=8.0 \mathrm{~Hz}, 2 \mathrm{H}), 7.89(\mathrm{~s}, 4 \mathrm{H}), 7.79(\mathrm{~d}, \mathrm{~J}=7.8 \mathrm{~Hz}, 2 \mathrm{H}), 2.05(\mathrm{~s}, 4 \mathrm{H}), 1.06-0.88(\mathrm{~m}, 20 \mathrm{H}), 0.67(\mathrm{t}, \mathrm{J}$ $=6.9 \mathrm{~Hz}, 6 \mathrm{H}), 0.48(\mathrm{~s}, 4 \mathrm{H}) .{ }^{13} \mathrm{C}$ NMR $(101 \mathrm{MHz}, \mathrm{DMSO}) \delta 163.61,153.32,151.87,146.53,141.29$, $134.47,131.76,125.35,121.28,120.38,116.43,98.14,55.25,40.15,31.06,28.89,28.34,23.15$, $21.95,13.75$.

\section{Conclusions}

In conclusion, the target compound $\mathbf{5}$ has been successfully prepared via the Knevenagel reaction in acceptable yields with a much milder and more efficient route, were designed to construct novel donor-acceptor conjugated organic molecules based on central fluorene units as electronic donors and cyanoacetic acid terminal groups as electronic acceptors.Optical properties, electrical Properties and thermodynamic properties will be studied in future work.

\section{Acknowledgements}

We are grateful for the financial support of the National Natural Science Foundation of China (No. 21462018), the Science Fund of the Technology Office of Jiangxi, China (20122BAB203017 and 20132BBE50024).

\section{Reference}

[1]. (a) Zhang, X. H.; Chen, B. J.; Lin, X. Q.; Wong, O. Y.; Lee. C. S.; Kwong, H. L.; Lee, S. T.; Wu, S. K. Chem. Mater. 2001, 13, 1565-1569. (b) Li, J. Y.; Liu, D.; Hong, Z. R.; Tong, S. W.; Wang, P. F.; Ma, C. W.; Lengyel, O.; Lee, C. S.; Kwong, H. L.; Lee, S. T. Chem. Mater. 2003, 15, 1486-1490.

[2]. Loi, M. A.; Denk, P.; Hoppe, H.; Neugebauer, H.; Winder, C.; Meissner, D.; Brabec , C.; Sariciftci, N. S.; Gouloumis, A.; Vazquezb, P.; Torresb, T. J. Mater. Chem. 2003, 13, 700-704.

[3]. (a)Li, Y. W.; Guo, Q.; Li, Z. F.; Pei, J. N.; Tian, W. J. Energy Environ. Sci. 2010, 3, 1427. (b)Gustafsson, G.; Gao, Y.; Treacy, G. M.; Klavetter, F.; Colaneri, N.; Heeger, A. J. Nature 1992,357, 477. (c) Roncali, J.; Frere, P.; Blanchard, P.; Bettignies, R.; Turbiez, M.; Roquet, , S.; Leriche, P.; Nicolas, Y. Thin Solid Films 2006, 511/512, 567.

[4]. Huo, Y. P.; Fang, X. M.; Huang, B. H.; Zhang, K. N. New Progress of Researches in Fluorene Compounds. Chin. J. Org. Chem. 2012, 32, 1169-1185. 
[5]. TANG, Z.; SUN, X.; YAN, H.; MIAO, L.. Optical properties of 5,5'-(9,9-dioctyl- 9Hfluorene-2,7-diyl)bis(thiophene-2-carbal dehyde): a novel organic light emitting material. Advanced Materials Research. 2014, 941-944, 662-665.

[6]. Chen, C. H.; Tang, C. W. Appl. Phys. Lett. 2001, 79, 3711. (b)Thomas, K. R. J.; Lin, J. T.; Tao, Y.T.; Chuen, C. H. Chem. Mater. 2002, 14, 3852. (c) Goes, M.;Verhoeven, J. W.; Hofstraat, H.;Brunner, K. Chem. Phys. Chem 2003, 4, 349. (d) Kulkarni, A. P.; Tonzola, C. J.; Babel, A.; Jenekhe, S. A. Chem. Mater. 2004,16, 4556. (e) Yan, H.; Lee, P.; Armstrong, N. R.; Graham, A.; Evmenenko, G.A.; Dutta, P.; Marks, T. J. J. Am. Chem. Soc. 2005, 127, 3172.

[7]. Pucher, N., A. Rosspeintner, V. Satzinger, V. Schmidt, G. Gescheidt, J. Stampfl and R. Liska. Structure-activity relationship in D- $\pi$-A- $\pi$-D-based photoinitiators for the two-photon-induced photopolymerization process. Macromolecules. 2009, 42, 6519-6528.

[8]. Li, Z. Q., M. Siklos, N. Pucher, K. Cicha, A. Ajami, W. Husinsky, A. Rosspeintner, E.Vauthey, G. Gescheidt, J. Stampfl and R. Liska. Synthesis and structure-activity relationship of several aromatic ketone-based two-photon initiators. J. Polym. Sci. A Polym. Chem. 2011, 49, 3688-3699.

[9]. He, G. S., L.-S. Tan, Q. D. Zheng and P. N. Prasad. Multiphoton absorbing materials: Molecular designs, syntheses, characterizations, and applications.Chem. Rev. 2008, 108, 1245-1330.

[10]. TANG, Z.; SUN, X.; MIAO, L.; SHEN, H.. Synthesis of a novel donor unit for organic light-emitting materials: 10-octyl-3,7-di(thiophen-2-yl)-10H-phenothiazine. Advanced Materials Research. 2014, 989-994, 284-287

[11]. Loi, M. A.; Denk, P.; Hoppe, H.; Neugebauer, H.; Winder, C.; Meissner, D.; Brabec , C.; Sariciftci, N. S.; Gouloumis, A.; Vazquezb, P.; Torresb, T. J. Mater. Chem. 2003, 13, 700-704.

[12]. Sun, X. B.; Liu, Y. Q,; Xu, X. J,; Yang, C.H,; Yu, G,; Chen, S. Y,; Zhao, Z.H,; Qiu, W. F,; Li, Y. F,; Zhu, D. B,; J. Phys. Chem. B, 2005,109, 21.

[13]. Zhou H, Zhou F, Tang S, et al. Dyes and Pigments, 2012, 92, 633-641.

[14]. Tacca A, Po R, Caldararo M, et al. Electrochimica acta, 2011, 56, 6638-6653.

[15]. Yang, C,; Song, H, S,; Liu, D, B.J Mater Sci, 2012, 47,3315 - 3319. 